\title{
Previsão top-down ou bottom-up? Impacto nos níveis de erro e de estoques de segurança
}

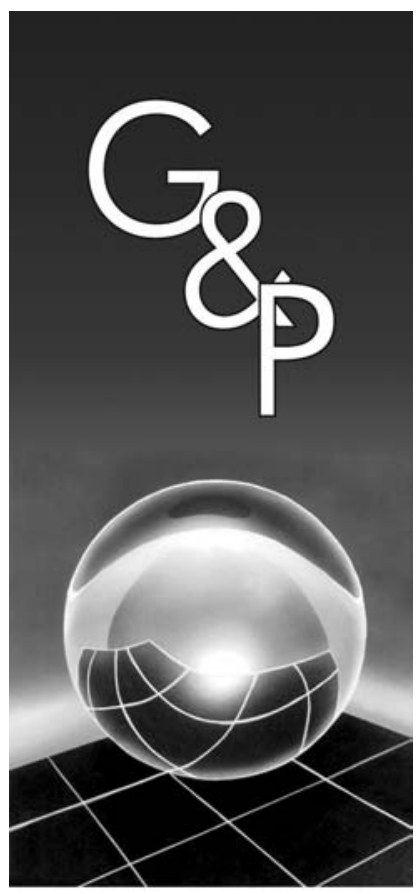

Peter Wanke

Resumo

A literatura de operações permanece sem concluir sobre a melhor abordagem em previsão de vendas: se da previsão dos dados agregados para os itens individuais (denominada abordagem top-down), ou se dos itens individuais para os dados agregados (denominada bottom-up). Nesse manuscrito são apresentados os resultados para a média e a variância dos erros de previsão com amortecimento exponencial nessas abordagens, considerando-se o caso em que a fração da série individual nas vendas agregados é estocástica. Por meio da geração de números aleatórios, são confirmados dois principais impactos na variância do erro de previsão, apresentados em pesquisas anteriores: efeitos portfólio e ancoragem. Os impactos também são avaliados em termos dos erros de previsão e do dimensionamento de estoques de segurança.

Palavras-chave: Abordagem de previsão. Amortecimento exponencial. Erros de previsão. Estoques de segurança.

\section{Introdução}

O objetivo desse artigo é analisar o comportamento do valor esperado e da variância dos erros de previsão de vendas nas abordagens top-down e bottom-up para identificar sob quais condições uma abordagem seria preferível à outra em termos de menores erros de planejamento e de menores níveis de estoque de segurança em operações de produção e distribuição. Além de constituírem diferentes modus operandi para se chegar a uma determinada previsão, deve ser lembrado que dentro das organizações cada uma das abordagens pode, individualmente, ser mais aderente a um determinado horizonte de planejamento e/ou tipo de tomada de decisão. Por exemplo, a abordagem top-down tende a ser empregada em horizontes de tempo mais longos e para dados mais agregados, ao passo que a abordagem bottom-up tende a ser mais adotada em horizontes de tempo mais curtos e para itens individuais.

Em estudos anteriores foram conduzidas análises com o método do amortecimento exponencial a partir de séries de vendas reais, chegando-se a diferentes conclusões sobre a adequação dessas abordagens para diferentes níveis de coeficiente de correlação, de variância das vendas e de participação (fração) da série individual nas vendas totais ou agregadas. Em outro estudo, foi apresentada a solução analítica para a variância do erro na abordagem top-down, supondo-se essa participação constante ao longo do tempo. Nele foram identificados dois fatores principais que podem favorecer a abordagem top-down em detrimento da bottom-up: efeito portfólio e efeito ancoragem.

Nessa pesquisa, foram gerados dados aleatórios de vendas, com base na distribuição normal bivariada, para se avaliar a adequação dessas abordagens quando a participação da série oscila ao redor da média, ou seja, quando apresenta um comportamento estocástico. Os resultados demonstram que a solução analítica apresentada na literatura para a variância da abordagem top-down fornece resultados mais precisos apenas quando a constante de amortecimento é pequena, mas não necessariamente igual a zero, e a previsão da participação é feita com a constante de amortecimento igual a zero. Também foi confirmado que a abordagem top-down é preferível à bottom-up para dimensionamento de estoques de segurança (menor variância do erro e pequeno viés) quando a correlação é baixa, a variância da série é relativamente pequena quando comparada à variância dos demais itens 
e a série possui pequena participação nas vendas agregadas. Os ganhos médios estimados oscilam ao redor de $3,2 \%$.

\section{Revisão de literatura}

\subsection{Abordagens top-down e bottom-up}

Existe grande consenso entre os autores sobre a conceituação e a operacionalização das abordagens top-down (TD) e bottom-up (BU) na previsão de vendas. Por exemplo, segundo Lapide (1998), na abordagem TD a previsão de vendas é feita para a soma de todos os itens, sendo então desagregada item a item, geralmente com base no percentual histórico do item no total. Nesse sentido, Schwarzkopf et al. (1988) apontam que na abordagem TD primeiramente é previsto o total agregado e posteriormente é feito o rateio com base em suas proporções históricas. Já na abordagem BU, cada um dos itens é previsto separadamente e as previsões são somadas caso seja necessária uma previsão agregada para o grupo (LAPIDE, 1998). Em outras palavras, na abordagem BU o previsor primeiro prepara as previsões para cada item, agregando-as em seguida no nível de interesse da análise (JAIN, 1995).

Autores como Gordon et al. (1997) e Gelly (1999), por exemplo, têm tentado relacionar a adequação das abordagens TD e BU a diferentes características das séries de vendas como, por exemplo, coeficiente de correlação entre o item em estudo e os demais itens agregados $(\rho)$; a fração ou proporção do item em estudo nas vendas totais agregadas (f); e a razão entre as variâncias dos demais itens agrupados e a do item em estudo $\left(\mathrm{k}^{2}\right)$. Uma premissa implícita em alguns dos artigos pesquisados está relacionada ao efeito portfólio, conceito definido inicialmente por Zinn et al. (1989) para avaliar o impacto da centralização dos estoques na variância das vendas agregadas de diferentes mercados.

De acordo com o efeito portfólio, a agregação das vendas minimiza a variância total quando o coeficiente de correlação das vendas entre os mercados é -1 e a razão entre as variâncias das vendas dos mercados é 1. Em linhas gerais, o racional para a redução da variância, segundo os autores, é a compensação das utuações das vendas entre dois mercados: quando as vendas de um mercado crescem, as vendas do outro mercado diminuem da mesma quantidade. Além disso, quando as variâncias possuem mesma magnitude, essa compensação tende a ocorrer com elevado grau de compatibilidade, por exemplo, sem sobras ou faltas de estoque. Esses efeitos podem ser inferidos a partir da soma das variâncias de duas variáveis aleatórias e são apresentados em maiores detalhes na seção 2.3.

Um exemplo nesse sentido é o artigo de Kahn (1998), segundo o qual, na abordagem TD os picos e vales carac- terísticos de cada item são cancelados pela agregação. A correlação negativa entre os itens reduziria a variância das vendas agregadas. Também corroborando as principais conclusões do efeito portfólio, Schwarzkopf et al. (1988) apontaram que estimativas baseadas em dados agregados são mais precisas que estimativas baseadas em previsões individuais quando os itens possuem padrões de vendas independentes (correlação nula).

Lapide (1998), entretanto, analisa a questão da correlação tendo como pano de fundo as tendências de crescimento e/ou decrescimento dos diferentes itens, que podem alterar suas proporções relativas ao longo do tempo. Proporções que crescem ou diminuem ao longo do tempo podem reduzir os benefícios da consolidação de itens com correlação negativa. Nesse caso, o autor afirma que, como regra geral, a abordagem TD só faz sentido se, e somente se, os padrões de vendas de cada item são os mesmos. Em outras palavras, se todos os itens estão crescendo, decrescendo ou se estão permanecendo estáveis, caracterizando correlação positiva entre as vendas dos diferentes itens. Por outro lado, o autor prossegue afirmando que, freqüentemente, uma família de produtos é composta por itens que potencialmente se canibalizam, como no caso de uma família com produtos novos e antigos. Para esses itens, o padrão de demanda é bastante diferente, já que alguns itens crescem à custa de outros (correlação negativa), o que tornaria a abordagem BU preferível.

Gordon et al. (1997) e Gelly (1999) discorrem sobre a adequação das abordagens TD e BU a outras características das séries de vendas. Mais especificamente, esses autores estudaram mais de 15.000 séries de vendas, de modo agregado e desagregado, gerando previsões com amortecimento exponencial triplo. A abordagem BU resultou em previsões mais precisas em $75 \%$ das séries, sendo os maiores ganhos de precisão obtidos para itens com forte correlação positiva e quando o mesmos representavam uma grande proporção da série agregada de vendas. Em contraste, quando os dados são negativamente correlacionados, a abordagem TD mostrou-se mais acurada, independentemente da participação do item na série agregada de vendas.

Finalmente, no estudo de caso apresentado por Gelly (1999), a abordagem TD mostrou-se mais adequada para itens que possuem um padrão de vendas previsível ao longo do tempo, por exemplo, com um pequeno coeficiente de variação nas vendas $(\mathrm{CV})$. Esse pequeno $\mathrm{CV}$ que poderia ser resultado simultâneo de uma grande participação do item na série agregada de vendas e de uma grande razão entre a variância da série de vendas dos demais itens agregados e a variância das vendas do item. Na Tabela 1 são resumidas as características que favorecem as abordagens TD e BU. 
Tabela 1. Características que favorecem as abordagens TD e BU.

\begin{tabular}{|c|c|c|}
\hline Autor & Top-down & Bottom-up \\
\hline Kahn (1998) & Correlação negativa $(\rho)$ & \\
\hline Lapide (1998) & $\begin{array}{l}\text { Correlação positiva, se as demandas apresentam mesma tendên- } \\
\text { cia de crescimento }\end{array}$ & $\begin{array}{l}\text { Correlação negativa, se as demandas } \\
\text { apresentam tendências inversas }\end{array}$ \\
\hline Gelly (1999) & $\begin{array}{l}\text { Pequeno coeficiente de variação (cv) } \\
\text { Grande participação no total (f) } \\
\text { Grande razão entre a variância dos demais itens agregados e a } \\
\text { da série de vendas }(\mathrm{k})\end{array}$ & \\
\hline Gordon et al. (1997) & $\begin{array}{l}\text { Correlação negativa } \\
\text { Qualquer participação no total }\end{array}$ & $\begin{array}{l}\text { Correlação positiva } \\
\text { Grande participação no total }\end{array}$ \\
\hline Schwarzkopf (1988) & Correlação nula & \\
\hline
\end{tabular}

\subsection{Amortecimento exponencial simples}

Segundo Gijbels, Pope e Wand (1999), o amortecimento exponencial simples (AES) é o modelo mais comumente utilizado na previsão de séries temporais. Suas principais vantagens são relacionadas ao fato de ser um modelo não paramétrico (ou seja, não associado a uma determinada distribuição de probabilidade), baseado em uma fórmula algébrica simples, que permite rapidamente a atualização da estimativa de nível local da série temporal através das recorrências em sua equação. Segundo Chatfield, Koehler e Snyder (2001), o AES e suas extensões foram desenvolvidos a partir do final dos anos 50 por Brown, Winters e Holt, dentre outros autores. Dentre suas principais premissas ou limitações, cabe destacar que no AES não são consideradas eventuais tendências de crescimento ou decrescimento, utuações sazonais e variações cíclicas.

Por exemplo, a previsão de vendas pelo AES para uma variável aleatória $\mathrm{X}$ é dada pela Equação 1:

$$
\mathrm{PX}_{\mathrm{t}}=\alpha^{*} \mathrm{X}_{\mathrm{t}-1}+(1-\alpha) * \mathrm{PX}_{\mathrm{t}-1}
$$

Em que $\mathrm{PX}_{\mathrm{t}}$ é a previsão de $\mathrm{X}$ para o período t; $\mathrm{X}_{\mathrm{t}-1}$ é a venda real de $\mathrm{X}$ em $\mathrm{t}-1$; e PXt ${ }_{-1}$ é a previsão de $\mathrm{X}$ em $\mathrm{t}-1$;

$\alpha$ é a constante de amortecimento, que varia entre 0 e 1 .

Analogamente, pode-se determinar a previsão da participação de $X$ nas vendas totais $T$ por meio da fração $\mathrm{f}(\mathrm{X} / \mathrm{T})$ com o AES (Equação 2):

$$
\mathrm{Pf}_{\mathrm{t}}=\beta * \mathrm{f}_{\mathrm{t}-1}+(1-\beta) * \mathrm{Pf}_{\mathrm{t}-1}
$$

Em que $\beta$ é a constante de amortecimento.

\subsection{Valor esperado e variância dos erros de previsão: o caso para f constante}

Wanke (2006) determinou as soluções analíticas para o valor esperado e a variância dos erros de previsão com AES nas abordagens TD e BU. Foram consideradas diferentes condições de tempo de resposta (determinístico ou estocástico) e a fração (f) das vendas do produto A nas vendas totais foi suposta constante. Especificamente, para o caso em que o tempo de resposta é determinístico e igual a 1 e sendo: a) $\mathrm{A}=$ variável aleatória que representa as vendas do produto A;

b) B = variável aleatória que representa as vendas agregadas dos demais produtos (exclusive $\mathrm{A}$ );

c) $\mathrm{T}=\mathrm{A}+\mathrm{B}-$ variável aleatória que representa as vendas totais agregadas;

d) $\mu \mathrm{A}=$ valor esperado de $\mathrm{A}$;

e) $\mu \mathrm{B}=$ valor esperado de $\mathrm{B}$;

f) $\mu \mathrm{T}=$ valor esperado de $\mathrm{T}$;

g) $\sigma \mathrm{A}=$ desvio padrão de $\mathrm{A}$;

h) $\mathrm{V}(\mathrm{A})=$ variância de $\mathrm{A}$;

i) $\sigma \mathrm{B}=$ desvio padrão de $\mathrm{B}$;

j) $V(B)=$ variância de $B$;

k) $\rho \mathrm{AB}=$ coeficiente de correlação entre $\mathrm{A}$ e $\mathrm{B}$;

l) $\sigma \mathrm{T}=\sqrt{\sigma \mathrm{A}^{2}+\sigma \mathrm{B}^{2}+2 * \rho \mathrm{AB} * \sigma \mathrm{A}^{*} \sigma \mathrm{B}}$ - desvio padrão de $\mathrm{T}$;

$\mathrm{m}) \mathrm{V}(\mathrm{T})=$ variância de $\mathrm{T}$;

n) $\mathrm{k}=\sigma \mathrm{B} / \sigma \mathrm{A}$ - razão entre os desvios padrão das variáveis aleatórias $\mathrm{A}$ e $\mathrm{B} ; \mathrm{e}$

o) $\mathrm{E}(\mathrm{f})=\mu \mathrm{A} / \mu \mathrm{T}$ - fração das vendas do produto $\mathrm{A}$ nas vendas totais agregadas $T$.

Foi demonstrado pelo mesmo autor que o erro médio de previsão em ambas as abordagens tende a zero para um número muito grande de dados históricos, ou seja, ambas as abordagens não implicam viés de previsão de vendas e o valor esperado da previsão em ambas as abordagens tende a $\mu \mathrm{A}$. Além disso:

a) na abordagem $\mathrm{BU}$, a variância da previsão de $\mathrm{A}$ tende a $\alpha^{*} \mathrm{~V}(\mathrm{~A}) /(2-\alpha)$ e a variância do erro de previsão de $\mathrm{A}$ tende a $2 * \mathrm{~V}(\mathrm{~A}) /(2-\alpha)$, para um número muito grande de dados históricos;

b) na abordagem TD, a variância da previsão de $\mathrm{A}$ tende a $\alpha * \mathrm{E}(\mathrm{F})^{2 *} \mathrm{~V}(\mathrm{~T}) /(2-\alpha)$ e a variância do erro de previsão de $\mathrm{A}$ tende a $\mathrm{V}(\mathrm{A})+\alpha^{*} \mathrm{E}(\mathrm{f}) 2 * \mathrm{~V}(\mathrm{~T}) /$ $(2-\alpha)$; e

c) o valor crítico de $\mathrm{k}$ que iguala as variâncias dos erros em ambas as abordagens é (Equação 3):

$$
\mathrm{k}=\frac{1-\mathrm{E}(\mathrm{f})^{2}}{\mathrm{E}(\mathrm{f}) *\left(\rho A B^{*} \mathrm{E}(\mathrm{f})+\sqrt{\mathrm{E}(\mathrm{f})^{2} *\left(\rho A B^{2}-1\right)+1}\right)}
$$


De acordo com os resultados apresentados por Wanke (2006), quanto menores os valores de $\rho \mathrm{AB}$ e $\mathrm{E}(\mathrm{f})$, maiores as chances de a abordagem TD minimizar a variância do erro de previsão. O efeito portfólio explica parte do resultado: um produto com pequena participação no total, negativamente correlacionado com as vendas agregadas dos demais produtos, apresenta menor variância do erro de previsão na abordagem TD por causa do cancelamento de parte de sua variância individual com a variância agregada dos demais itens.

Mesmo para valores mais elevados de $\rho \mathrm{AB}$ e $\mathrm{E}(\mathrm{f})$, a abordagem TD pode apresentar melhores resultados que a BU se o valor de $\mathrm{k}$ for suficientemente baixo, ou seja, se $\sigma \mathrm{A}$ for suficientemente maior que $\sigma \mathrm{B}$. Esse resultado seria totalmente explicado pelo efeito ancoragem (dado que não se verifica o efeito portfólio): à variância do item A, seria apropriada uma parcela menos que proporcional da variância total (visto que o termo $\alpha^{*} \mathrm{E}(\mathrm{f})^{2} /(2-\alpha)$ é necessariamente menor que 1). Na Figura 1, os Efeitos Portfólio e Ancoragem são ilustrados com exemplos numéricos.

Por fim, deve ser apontado que para $\alpha=0$, as variâncias dos erros de previsão das abordagens TD e BU são minimizadas e correspondem a V(A). Também deve ser considerado que o valor esperado do erro de previsão em ambas as abordagens é zero. Neste momento, cabe a pergunta: se $\alpha=0$ minimiza a variância do erro de previsão e se seu valor esperado é zero para séries de dados suficientemente longas (ou seja, para o longo prazo em séries com regime estacionário), para que pesquisar ou buscar um valor de $\alpha$ entre 0 e 1 de modo a minimizar o erro de previsão, procedimento adotado na prática, segundo Silver et al. (2002)?

Esse procedimento decorre do erro amostral intrínseco aos sistemas de planejamento de vendas e estoques das empresas. Quando todos os dados históricos (ou um número suficientemente grande de observações) estão disponíveis, estimativas estatísticas para a média e o desvio padrão podem ser calculadas com a significância desejada. Inferências sobre a distribuição de probabilidade da variável aleatória "vendas" também podem ser feitas. No entanto, muitos sistemas empresariais não utilizam ou possuem todos os dados históricos, o que gera dúvidas se os erros de previsão resultam de ruídos aleatórios ou de uma mudança real em um ou mais parâmetros da distribuição das vendas. Infelizmente, a priori não é possível esclarecer essas dúvidas sobre as vendas reais, mas somente a posteriori (SILVER et al., 2002). Daí a busca de um valor de $\alpha$ ótimo que minimize esses ruídos aleatórios.

De qualquer forma, escolhas devem ser feitas com relação ao valor de $\alpha$ e aos pesos atribuídos aos dados passados. Maiores valores de $\alpha$ implicam maior peso às observações recentes, e menores valores de $\alpha$ incorporam

Efeito portfólio e efeito ancoragem combinados:

$\sigma A=10 ; \sigma B=20 ; E(f)=0,30 ; \rho A B=-0,40 ; \alpha=0,10$

Variância $B U=2^{*} \sigma A^{2} /(2-\alpha)=2100 /(2-0,10)=105,26$

Variância TD $=\sigma A^{2}+\left[\alpha^{\star} E(f)^{2} /(2-\alpha)\right]^{\star} \sigma T^{2}$

Variância TD $=100+\left[0,10^{*} 0,30^{2} /(2-0,10)\right]^{*}\left[10^{2}+20^{2}+2 * 10 * 20 *(-0,40)\right]$

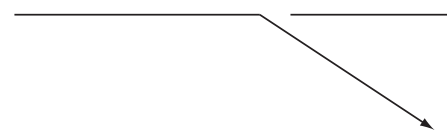

Ao efeito portfólio se complementa o

efeito ancoragem, sendo incorporada a

variância total menos que proporcional à

do efeito portfólio (o termo $\alpha^{\star} \mathrm{E}(\mathrm{f})^{2} /(2-\alpha)$

Variância TD = $100+1,61=101,61$ é menor que 1).

Ou seja, tem-se que: $\mathrm{k}=0,5$ e $\mathrm{k}_{\text {crítico }}=0,28$, o que torna preferível a abordagem TD.

Apenas efeito ancoragem:

$\sigma \mathrm{A}=10 ; \sigma \mathrm{B}=2 ; \mathrm{E}(\mathrm{f})=0,80 ; \rho \mathrm{A}=+0,40 ; \alpha=0,10$

Variância $B U=2 * \sigma A^{2} /(2-\alpha)=2100 /(2-0,10)=105,26$

Variância $T D=\sigma A^{2}+\left[\alpha^{\star} E(f)^{2} /(2-\alpha)\right] . \sigma T^{2}$

Variância TD $=100+\left[0,10 * 0,80^{2} /(2-0,10)\right] *\left[10^{2}+20^{2}+2 * 10 * 20 *(-0,40)\right]$

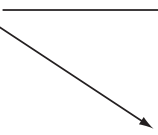

Variância TD $=100+4,04=104,04$
A variância total (120) é menor que a soma das variâncias de A e B (104) pela correlação positiva. Não há efeito portfólio.
No entanto, é incorporada à variância de A uma parcela da variância total menos que proporcional à da variância total $\left(\alpha^{*} E(f)^{2} /(2-\alpha)\right)$, pelo efeito ancoragem.

Ou seja, tem-se que: $k=5$ e $k_{\text {critico }}=2,22$, o que torna preferível a abordagem TD.

Figura 1. Efeitos portfólio e ancoragem. 
uma maior parcela dos dados históricos. Se as vendas podem ser descritas de fato como uma variável aleatória, a minimização dos erros de previsão através de elevados valores de $\alpha$ deve ser vista com cuidado. Segundo os autores, elevados valores de $\alpha$ (superiores a 0,3 ) devem levantar dúvidas sobre a validade do modelo de nível constante para descrever o comportamento aleatório das vendas. Elevadas constantes de amortecimento indicam a presença de um componente de tendência na série de vendas. Os autores sugerem trabalhar com valores de $\alpha$ entre 0,01 e 0,30 com um valor de 0,10 , sendo bastante razoável para séries sem tendência de crescimento.

Nestas circunstâncias amostrais, a melhor estimativa da média de vendas é simplesmente a previsão de vendas para o próximo período. No entanto, para estimar a variância das vendas, deve ser utilizada a variância do erro de previsão (SILVER; PETERSON, 1985). Ainda segundo Greene (1997), a variância do erro de previsão e a variância das vendas não são iguais. De acordo com esses autores, uma maior adequação da variância do erro de previsão no lugar da variância da demanda está relacionada à utilização da previsão para estimar as vendas. $\mathrm{O}$ estoque de segurança, por exemplo, deveria ser dimensionado para proteger contra variações nos erros de previsão de vendas.

Em resumo, percebe-se que a variância do erro de previsão de vendas pode ser in uenciada por diferentes fatores, que são: (1) a abordagem de previsão adotada e sua adequação a diferentes características da série vendas (conforme aquelas apresentadas na Tabela 1 e na Figura 1); (2) a premissa adotada com relação à fração f para análise da série (se determinística ou estocástica); e (3) o erro amostral presente nos sistemas de planejamento e que pode ser atenuado por constantes de amortecimento diferentes de zero. Torna-se relevante, portanto, compreender como esses fatores podem levar em conjunto a uma menor variância do erro de previsão de vendas e, consequentemente, identificar oportunidades para redução dos níveis de estoque de segurança. Conforme será visto a seguir, os fatores (2) e (3) serão analisados no âmbito da abordagem de previsão mais adequada para o método do AES.

\section{Objetivos e metodologia da pesquisa}

Considerando a revisão de literatura, são apresentadas as principais questões a serem respondidas pela pesquisa:

a) qual o comportamento do valor esperado e da variância dos erros de previsão de vendas na abordagem TD quando f é estocástico (variável)?

b) em que medida considerar o valor de $\mathrm{f}$ constante favorece determinada abordagem? Sob quais condições essa premissa é uma boa aproximação? c) em quais circunstâncias são verificados os Efeitos Portfólio e Ancoragem quando f é estocástico? Qual o impacto sobre a ocorrência do efeito portfólio e ancoragem nas abordagens TD e BU quando f é estocástico? e

d) quais as implicações acadêmicas e gerenciais desses resultados para a previsão de vendas e o dimensionamento de estoques de segurança?

Para se atingir esses objetivos, são adotadas duas abordagens metodologicamente complementares: modelagem analítica do valor esperado e da variância dos erros de previsão, a partir dos trabalhos seminais de Goodman (1960) e Bohrnstedt e Goldberger (1969), e geração de valores aleatórios via simulação. Conforme é discutido na seção seguinte em maiores detalhes, a geração de valores aleatórios se torna necessária por que f apresenta distribuição semelhante à de Cauchy e suas integrais para o valor esperado e variância não convergem para uma formulação analítica.

\section{Metodologia}

\subsection{Modelagem analítica}

Quando f é variável, o cálculo da variância dos erros de previsão na abordagem TD é alterado pela introdução de correlação (covariância) entre a fração $f$ e as vendas totais $\mathrm{T}$ e pela necessidade de se prever o valor da fração f para o período seguinte, de modo a se obter a previsão de vendas para o produto A, na forma (Equação 4):

$$
\mathrm{PA}=\mathrm{PT}^{*} \mathrm{Pf}
$$

Em que:

e) $\mathrm{PA}=$ previsão de vendas para o produto A no período seguinte;

f) $\mathrm{PT}=$ previsão de vendas para as vendas totais $\mathrm{T}$ no período seguinte; e

g) $\mathrm{Pf}$ = previsão da fração de $\mathrm{A} / \mathrm{T}$ no período seguinte.

Nesse modelo, o erro de previsão para o produto A na abordagem TD é dado pela Equação 5:

$$
\mathrm{EA}=\mathrm{A}-\mathrm{PA}
$$

Em que:

h) $\mathrm{EA}=$ erro de previsão do produto $\mathrm{A}$ no período seguinte; e

i) A = variável aleatória que representa as vendas reais de A.

Tomando-se os valores esperados, tem-se a Equação 6:

$$
\mathrm{E}(\mathrm{EA})=\mathrm{E}(\mathrm{A})-\mathrm{E}(\mathrm{PA})
$$

Mas (Equação 7),

$$
\mathrm{E}(\mathrm{PA})=\mathrm{E}(\mathrm{PT} * \mathrm{Pf})=\mathrm{COV}(\mathrm{PT}, \mathrm{Pf})+\mathrm{E}(\mathrm{PT}) * \mathrm{E}(\mathrm{Pf})(7)
$$


De acordo com Wanke (2006); $\mathrm{E}(\mathrm{PT})=\mathrm{E}(\mathrm{T})$ e $\mathrm{E}(\mathrm{Pf})=\mathrm{E}(\mathrm{f})$.

Logo (Equação 8):

$$
\mathrm{E}(\mathrm{PA})=\mathrm{COV}(\mathrm{PT}, \mathrm{Pf})+\mathrm{E}(\mathrm{T}) * \mathrm{E}(\mathrm{f})
$$

Sendo que COV(PT, Pf) representa a covariância entre a previsão de $\mathrm{T}$ e a previsão de f, ou seja, é o viés esperado da previsão de vendas do produto A pela abordagem TD. Dessa forma (Equação 9):

$$
\mathrm{E}(\mathrm{EA})=\operatorname{COV}(\mathrm{T}, \mathrm{f})-\operatorname{COV}(\mathrm{PT}, \mathrm{Pf})
$$

Em que $\operatorname{COV}(T, f)$ representa a covariância entre $T$ e f. Ou seja, o erro médio de previsão de vendas do produto A, na abordagem TD quando f é variável, apresenta viés dado pela diferença entre as covariâncias de $\mathrm{T}$ e $\mathrm{f}$ e de PT e Pf. Calculando-se as variâncias, tem-se que (Equação 10):

$$
\mathrm{V}(\mathrm{EA})=\mathrm{V}(\mathrm{A})+\mathrm{V}(\mathrm{PA})
$$

Mas (Equação 11),

$$
\mathrm{V}(\mathrm{PA})=\mathrm{V}(\mathrm{PT} * \mathrm{Pf})
$$

Goodman (1960) e Bohrnstedt e Goldberger (1969) desenvolveram o resultado exato para a variância do produto de duas variáveis aleatórias correlacionadas (por exemplo, T e f), de modo que (Equação 12):

$$
\begin{aligned}
& \mathrm{V}(\mathrm{PT} * \mathrm{Pf})=\mathrm{E}(\mathrm{T})^{2} * \mathrm{~V}(\mathrm{Pf})+\mathrm{E}(\mathrm{f})^{2} * \mathrm{~V}(\mathrm{PT})+ \\
& +\mathrm{V}(\mathrm{PT}) * \mathrm{~V}(\mathrm{Pf})+2 * \mathrm{E}(\mathrm{f}) * \mathrm{COV}\left(\Delta P T^{2}, \Delta \mathrm{Pf}\right)+ \\
& +2 * \mathrm{E}(\mathrm{T}) * \mathrm{COV}\left(\Delta \mathrm{PT}, \Delta \mathrm{Pf}^{2}\right)+ \\
& +2 * \mathrm{E}(\mathrm{f}) * \mathrm{E}(\mathrm{T}) * \operatorname{COV}(\mathrm{PT}, \mathrm{Pf})-\operatorname{COV}(\mathrm{PT}, \mathrm{Pf})^{2}+ \\
& +\operatorname{COV}\left(\Delta \mathrm{PT}^{2}, \Delta P f^{2}\right)
\end{aligned}
$$

Sendo que (Equações 13 e 14)

$$
\begin{gathered}
\mathrm{DPT}=\mathrm{PT}-\mathrm{E}(\mathrm{PT})=\mathrm{PT}-\mathrm{E}(\mathrm{T}) \\
\mathrm{DPf}=\mathrm{Pf}-\mathrm{E}(\mathrm{Pf})=\mathrm{PT}-\mathrm{E}(\mathrm{f})
\end{gathered}
$$

Mas, segundo Wanke (2006) (Equações 15 e 16),

$$
\begin{aligned}
& \mathrm{V}(\mathrm{PT})=\frac{\alpha}{2-\alpha} * \mathrm{~V}(\mathrm{~T}) \\
& \mathrm{V}(\mathrm{Pf})=\frac{\beta}{2-\beta} * \mathrm{~V}(\mathrm{f})
\end{aligned}
$$

Dessa forma, tem-se que a Equação 17:

$$
\begin{aligned}
& \mathrm{V}(\mathrm{PT} * \mathrm{Pf})=\frac{\beta}{2-\beta} * \mathrm{E}(\mathrm{T})^{2} * \mathrm{~V}(\mathrm{f})+\frac{\alpha}{2-\alpha} * \mathrm{E}(\mathrm{f})^{2} * \mathrm{~V}(\mathrm{~T})+ \\
& +\frac{\alpha * \beta}{(2-\alpha) *(2-\beta)} * \mathrm{~V}(\mathrm{~T}) * \mathrm{~V}(\mathrm{f})+ \\
& +2 * \mathrm{E}(\mathrm{f}) * \operatorname{COV}\left(\Delta P T^{2}, \Delta \mathrm{Pf}\right)+2 * \mathrm{E}(\mathrm{T}) * \operatorname{COV}\left(\Delta P T, \Delta \mathrm{Pf}^{2}\right)+ \\
& +2 * \mathrm{E}(\mathrm{f}) * \mathrm{E}(\mathrm{T}) * \operatorname{COV}(\mathrm{PT}, \mathrm{Pf})-\operatorname{COV}(\mathrm{PT}, \mathrm{Pf})^{2}+ \\
& +\operatorname{COV}\left(\Delta P T^{2}, \Delta \mathrm{Pf}^{2}\right)
\end{aligned}
$$

Finalmente, calculando-se as variâncias para o erro de previsão do produto $\mathrm{A}$ na abordagem $\mathrm{TD}$, tem-se a Equação 18:

$$
\begin{aligned}
& \mathrm{V}(\mathrm{EA})=\mathrm{V}(\mathrm{A})+\frac{\beta}{2-\beta} * \mathrm{E}(\mathrm{T})^{2} * \mathrm{~V}(\mathrm{f})+ \\
& +\frac{\alpha}{2-\alpha} * \mathrm{E}(\mathrm{f})^{2} * \mathrm{~V}(\mathrm{~T})+\frac{\alpha * \beta}{(2-\alpha)^{*}(2-\beta)} * \mathrm{~V}(\mathrm{~T}) * \mathrm{~V}(\mathrm{f})+ \\
& +2 * \mathrm{E}(\mathrm{f}) * \mathrm{COV}\left(\Delta P T^{2}, \Delta \mathrm{Pf}\right)+2 * \mathrm{E}(\mathrm{T}) * \mathrm{COV}\left(\Delta P T, \Delta \mathrm{Pf}^{2}\right)+ \\
& +2 * \mathrm{E}(\mathrm{f}) * \mathrm{E}(\mathrm{T}) * \mathrm{COV}(\mathrm{PT}, \mathrm{Pf})-\mathrm{COV}(\mathrm{PT}, \mathrm{Pf})^{2}+ \\
& +\mathrm{COV}\left(\Delta P T^{2}, \Delta \mathrm{Pf}^{2}\right)
\end{aligned}
$$

Ou seja, dependendo da magnitude e do sinal dos termos referentes às covariâncias é possível que a variância da abordagem TD seja menor que a BU sob condições específicas.

Ainda que seja possível reduzir os termos $\operatorname{COV}\left(\Delta \mathrm{PT}^{2}, \Delta \mathrm{Pf}\right), \quad \operatorname{COV}\left(\Delta \mathrm{PT}, \Delta \mathrm{Pf}^{2}\right), \quad \operatorname{COV}(\mathrm{PT}, \mathrm{Pf}) \quad \mathrm{e}$ $\operatorname{COV}\left(\Delta \mathrm{PT}^{2}, \Delta \mathrm{Pf}^{2}\right)$ a zero ou a expressões com o valor esperado, a variância e a covariância entre $\mathrm{T}$ e f quando $\alpha$ e $\beta$ são menores que 0,25 (cf. Anexo 1), não existe formulação analítica para o valor esperado e a variância da razão entre duas variáveis aleatórias, ou seja, para $\mathrm{E}(\mathrm{f})$ e $\mathrm{V}(\mathrm{f})$. Isso porque $\mathrm{f}$ apresenta distribuição semelhante à de Cauchy (WEISSTEIN, 1999; ZWILLINGER; KOKOSA, 2000) e as duas integrais divergem para os momentos de ordem maior que 1 (cf. Anexo 2).

\subsection{Geração de resultados via simulação em Excel}

Para geração dos experimentos em planilha Excel relacionados às abordagens TD e BU em previsão de vendas e coleta de suas principais estatísticas, considerou-se que A e $B$ seguem uma distribuição normal bivariada com médias $\mu \mathrm{A}$ e $\mu \mathrm{B}$, desvios padrão $\sigma \mathrm{A}$ e $\sigma \mathrm{B}$ e coeficiente de correlação dado por $\rho \mathrm{AB}$. De acordo com Kenney e Keeping (1951), se z1 e z2 são suas variáveis aleatórias independentes e normalmente distribuídas com média e desvio padrão, respectivamente, 0 e 1, A e B apresentam distribuição normal bivariada com coeficiente de correlação $\rho \mathrm{AB}$, se forem geradas da seguinte forma (Equações 19 e 20): 


$$
\begin{aligned}
& \mathrm{A}=\mu \mathrm{A}+\left(\frac{1+\rho \mathrm{AB}}{2}\right)^{1 / 2} * \sigma \mathrm{A} * \mathrm{z} 1+\left(\frac{1-\rho \mathrm{AB}}{2}\right)^{1 / 2} * \sigma \mathrm{A} * \mathrm{z} 2 \\
& \mathrm{~B}=\mu \mathrm{B}+\left(\frac{1+\rho \mathrm{AB}}{2}\right)^{1 / 2} * \sigma \mathrm{B} * \mathrm{z} 1-\left(\frac{1-\rho \mathrm{AB}}{2}\right)^{1 / 2} * \sigma \mathrm{B} * \mathrm{z} 2
\end{aligned}
$$

Sendo que, no Excel:

$\mathrm{z}=$ INV.NORM P(ALEATÓRIO( )).

Foram conduzidos três experimentos principais visando avaliar o impacto de diferentes condições da série de vendas nos erros das abordagens TD e BU, a saber:

a) coeficiente de correlação $\rho \mathrm{AB}$, com cinco níveis: $-1 ;-0,5 ; 0 ;+0,5$ e +1 ;

b) razão $\mathrm{k}$, com três níveis: 0,$5 ; 1$ e 2 ; e

c) mediana da fração $\mathrm{f}$, com três níveis: 0,$25 ; 0,50$ e 0,75 .

Todos os experimentos estão conectados pelo caso base $\mu \mathrm{A}=\mu \mathrm{B}=100 ; \sigma \mathrm{A}=\sigma \mathrm{B}=20 ; \rho \mathrm{AB}=0$ e $\mathrm{f}=0,50$. Para cada nível de cada um dos quatro experimentos foram realizadas 100 replicações, variando-se $\alpha$ e $\beta$ de 0 até 1 em iguais intervalos de 0,25 (total de $5 * 5 * 100=$ 2.500 replicações por nível por experimento). Cada replicação consistiu da geração de séries de vendas de A e B compostas por 10.000 dados, nas quais foram testadas as abordagens TD e BU e coletados os resultados. Para cada nível foram calculados os valores médios dos seguintes termos: E(PA), E(EA), V(PA), V(EA), E(f), V(f), $\operatorname{COV}(\mathrm{T}, \mathrm{f}), \operatorname{COV}(\mathrm{PT}, \mathrm{Pf}), \operatorname{COV}(\mathrm{T}, \mathrm{Pf}), \operatorname{COV}(\mathrm{PT}, \mathrm{f})$, $\operatorname{COV}\left(\mathrm{T}^{2}, \mathrm{f}^{2}\right), \quad \operatorname{COV}\left(\Delta \mathrm{PT}^{2}, \Delta \mathrm{Pf}\right), \quad \operatorname{COV}\left(\Delta \mathrm{PT}, \Delta \mathrm{Pf}^{2}\right) \quad \mathrm{e}$ $\operatorname{COV}\left(\Delta \mathrm{PT}^{2}, \Delta \mathrm{Pf}^{2}\right)$

Os resultados relativos à média e à variância dos erros dessas abordagens nesses três experimentos são analisados na próxima seção. Em sequiência, são comentadas as principais implicações para previsão de vendas (relativa ao tratamento do viés da previsão) e gestão de estoques (relativa à determinação dos estoques de segurança) na abordagem TD.

\section{Análise dos resultados}

\subsection{Impacto do coeficiente de correlação $\rho A B$}

$O$ impacto de $\rho A B$ na abordagem TD depende da combinação de valores de $\alpha$ e $\beta$. Se $\alpha>\beta$, menores valores de $\rho \mathrm{AB}$ implicam redução na variância do erro de previsão, sendo obtidos, simultaneamente, ganhos com o efeito portfólio e o efeito ancoragem. Se $\alpha<\beta$, maiores valores de $\rho \mathrm{AB}$ implicam redução na variância da abordagem TD, sendo apenas obtidos ganhos com o efeito ancoragem. Para $\alpha=\beta, \rho \mathrm{AB}$ não afeta a variância na abordagem $\mathrm{TD}$, igual à abordagem BU (Tabela 2).
Comparativamente à variância da abordagem BU, dado um par $(\alpha, \beta)$, a abordagem TD tende a ser preferível a BU quando são verificados simultaneamente os Efeitos Portfólio e Ancoragem, ou seja, quando $\rho \mathrm{AB}$ é negativo e $\alpha>\beta$. Em outras palavras, $\alpha>\beta$ significa que maior peso é dado às observações passadas de $\mathrm{f}$, comparativamente, às de T. O leitor deve lembrar que, no AES a constante de amortecimento atribui pesos decrescentes aos dados passados. Quanto mais próxima de 1 for essa constante, mais peso é atribuído aos dados mais recentes e menos peso aos dados mais antigos, ao passo que uma constante mais próxima de zero implica decremento suave dos pesos ao longo das observações passadas.

Comparativamente à variância da abordagem TD, os resultados gerados pela fórmula para $\mathrm{f}$ constante $\left(\mathrm{V}(\mathrm{A})+\alpha^{*} \mathrm{E}(\mathrm{f})^{2 *} \mathrm{~V}(\mathrm{~T}) /(2-\alpha)\right)$ apresentam valores exatos para os casos em que $\beta=0$. Quando $\rho \mathrm{AB}=+1$, essa fórmula também fornece os resultados exatos para a variância da abordagem TD para quaisquer valores de $\beta$. Ou seja, quando A e B têm correlação positiva perfeita, o impacto de suas utuações de mesmo sentido e magnitude se cancelam na fração f, anulando o erro amostral de utilizar parcialmente os dados históricos de f com peso decrescente (devido a um maior valor de $\beta$, que implica queda acelerada nos pesos atribuídos aos dados passados e, consequentemente, maior concentração dos mesmos nos dados mais recentes). Por outro lado, quando A e B têm correlação negativa perfeita $(\rho \mathrm{AB}=-1)$, suas utuações em sentidos opostos e de mesma magnitude se cancelam em $\mathrm{T}$, anulando o erro amostral associado a utilizar parcialmente os dados históricos de T com pesos decrescentes.

Finalmente, com relação ao erro médio de previsão (ou viés), verifica-se que para quaisquer valores de $\alpha$ e $\beta$, os mesmos são iguais a zero quando $\rho \mathrm{AB}=+1$ e $\rho \mathrm{AB}=-1$, tendendo a zero nos demais níveis.

\subsection{Impacto da razão $k$}

Os impactos da razão $\mathrm{k}$ na média e na variância do erro de previsão na abordagem TD dependem de $\alpha$ e $\beta$. Além disso, a fórmula para f constante fornece valores próximos para o caso de f estocástico, qualquer que seja o valor de $\alpha$, desde que $\beta=0$ e $\mathrm{k} \leq 1$ (Tabela 3 ).

Quando $\mathrm{k}<1$, maiores valores de $\alpha$ implicam aumento na variância e diminuição no erro médio, para quaisquer valores de $\beta$, exceto para $\beta$ igual a zero. O mesmo acontece com maiores valores de $\beta$, para quaisquer valores de $\alpha$, exceto quando $\alpha=0$. Para $\mathrm{k}=1$, maiores valores de $\alpha$, dado $\beta$ e vice-versa, implicam aumento na variância da abordagem TD e erro médio de previsão aproximadamente igual a zero. Já para $\mathrm{k}>1$, maiores valores de $\alpha$, dado um mesmo valor de $\beta$, implicam redução na variância na abordagem TD se $\alpha<\beta$. Por outro lado, maiores valores de $\beta$, dado $\alpha$, geralmente implicam aumento na variância se $\beta>\alpha$. Quando $k>1$, maiores valores de $\alpha$, 
Tabela 2. Resultados do experimento com $\rho \mathrm{AB}$.

\begin{tabular}{|c|c|c|c|c|c|c|c|c|c|c|c|c|}
\hline \multicolumn{2}{|c|}{ f estocástico } & \multicolumn{2}{|c|}{$\rho A B=-1$} & \multicolumn{2}{|c|}{$\rho A B=-0,5$} & \multicolumn{2}{|c|}{$\rho \mathbf{A B}=\mathbf{0}$} & \multicolumn{2}{|c|}{$\rho \mathbf{A B}=+\mathbf{0 , 5}$} & \multicolumn{2}{|c|}{$\rho \mathrm{AB}=+1$} & \multirow{2}{*}{$\begin{array}{c}\text { f constante } \\
\mathrm{V}(\mathrm{BU}) \\
\end{array}$} \\
\hline$\alpha$ & $\beta$ & E(EA) & $\mathbf{V}($ EA $)$ & $\mathbf{E}(\mathbf{E A})$ & V(EA) & E(EA) & $\mathbf{V}(\mathbf{E A})$ & E(EA) & $\mathbf{V}($ EA) & E(EA) & $\mathrm{V}(\mathbf{E A})$ & \\
\hline \multirow[t]{5}{*}{-} & - & - & 400,000 & - & 400,000 & - & 400,000 & - & 400,000 & - & 400,000 & 400,000 \\
\hline & 0,25 & - & 457,140 & 0,001 & 444,160 & $(0,002)$ & 430,470 & 0,001 & 415,800 & - & 400,000 & \\
\hline & 0,50 & - & 533,333 & $(0,000)$ & 503,180 & $(0,002)$ & 471,210 & 0,002 & 436,780 & - & 400,000 & \\
\hline & 0,75 & - & 640,000 & $(0,002)$ & 585,900 & $(0,005)$ & 528,550 & $(0,005)$ & 466,950 & - & 400,000 & \\
\hline & 1,00 & - & 800,000 & 0,001 & 709,200 & 0,003 & 613,000 & $(0,000)$ & 510,850 & - & 400,000 & \\
\hline \multirow[t]{5}{*}{0,25} & - & - & 400,000 & 0,000 & 414,290 & 0,001 & 428,570 & $(0,003)$ & 442,850 & - & 457,140 & 457,140 \\
\hline & 0,25 & - & 457,140 & 0,004 & 458,220 & $(0,002)$ & 458,630 & $(0,003)$ & 458,360 & - & 457,140 & \\
\hline & 0,50 & - & 533,333 & 0,002 & 516,480 & 0,002 & 498,690 & $(0,002)$ & 478,890 & - & 457,140 & \\
\hline & 0,75 & - & 640,000 & $(0,002)$ & 598,420 & 0,002 & 554,060 & 0,002 & 507,240 & - & 457,140 & \\
\hline & 1,00 & - & 800,000 & 0,001 & 720,960 & 0,003 & 637,630 & $(0,002)$ & 550,770 & - & 457,140 & \\
\hline \multirow[t]{5}{*}{0,50} & - & - & 400,000 & $(0,002)$ & 433,340 & $(0,022)$ & 466,700 & $(0,001)$ & 500,000 & - & 533,333 & 533,333 \\
\hline & 0,25 & - & 457,140 & 0,001 & 476,950 & $(0,003)$ & 496,600 & 0,004 & 515,430 & - & 533,333 & \\
\hline & 0,50 & - & 533,333 & $(0,000)$ & 534,960 & $(0,001)$ & 535,530 & 0,002 & 535,070 & - & 533,333 & \\
\hline & 0,75 & - & 640,000 & $(0,001)$ & 615,960 & 0,001 & 590,210 & $(0,003)$ & 563,150 & - & 533,333 & \\
\hline & 1,00 & - & 800,000 & 0,001 & 737,330 & $(0,000)$ & 672,540 & 0,001 & 604,610 & - & 533,333 & \\
\hline \multirow[t]{5}{*}{0,75} & - & - & 400,000 & $(0,004)$ & 460,000 & $(0,004)$ & 520,010 & 0,004 & 579,840 & - & 640,000 & 640,000 \\
\hline & 0,25 & - & 457,140 & 0,001 & 503,870 & $(0,004)$ & 550,300 & $(0,000)$ & 595,210 & - & 640,000 & \\
\hline & 0,50 & - & 533,333 & 0,000 & 561,250 & $(0,000)$ & 588,520 & 0,004 & 614,180 & - & 640,000 & \\
\hline & 0,75 & - & 640,000 & 0,004 & 641,520 & $(0,001)$ & 642,060 & $(0,001)$ & 641,360 & - & 640,000 & \\
\hline & 1,00 & - & 800,000 & $(0,000)$ & 761,880 & $(0,001)$ & 722,880 & 0,000 & 648,750 & - & 640,000 & \\
\hline \multirow[t]{5}{*}{1,00} & - & - & 400,000 & $(0,000)$ & 500,000 & 0,005 & 599,980 & $(0,001)$ & 700,000 & - & 800,000 & 800,000 \\
\hline & 0,25 & - & 457,140 & 0,002 & 543,920 & 0,002 & 630,010 & 0,002 & 715,360 & - & 800,000 & \\
\hline & 0,50 & - & 533,333 & $(0,002)$ & 601,090 & $(0,001)$ & 668,530 & 0,000 & 734,470 & - & 800,000 & \\
\hline & 0,75 & - & 640,000 & $(0,000)$ & 680,460 & 0,001 & 720,710 & $(0,001)$ & 760,460 & - & 800,000 & \\
\hline & 1,00 & - & 800,000 & - & 800,000 & - & 800,000 & - & 800,000 & - & 800,000 & \\
\hline
\end{tabular}

Valores negativos são indicados por parênteses.

dado $\beta$ e vice-versa, levam a reduções no erro médio de previsão, exceto quanto $\alpha=0$.

Comparativamente à variância na abordagem $\mathrm{BU}$, a variância na abordagem TD tende a ser menor quanto maior for a relação $\alpha / \beta$ e k $\leq 1$, indicando que o efeito ancoragem é relevante quando a variância total possui valor próximo da variância de A. Por outro lado, quando $\mathrm{k}>1$, para $\alpha>\beta$ e $\beta<0,50$, a variância na abordagem BU tende a ser menor que a variância na abordagem TD, qualquer que seja o valor de $\mathrm{k}$, não indicando que o efeito ancoragem seja relevante.

O principal impacto de uma razão $\mathrm{k}$ diferente de 1 está na introdução de um viés ou de um erro de previsão médio significativamente diferente de zero na abordagem TD. Para um dado valor de $\alpha$, maiores valores de $\beta$ reduzem a magnitude do viés, à exceção de $\alpha=0$. Por sua vez, dado um valor de $\beta$, maiores valores de $\alpha$ reduzem a magnitude do viés, à exceção de $\beta=0$. Há um trade-off entre o erro médio e sua variância na abordagem $\mathrm{TD}$, sobretudo para $\mathrm{k} \leq 1$, referente ao erro amostral de $\mathrm{T}$ e f. A escolha é entre incorporar apenas informações mais recentes sobre a posição de $\mathrm{T}$ e f (maiores $\alpha$ e $\beta$ ) para reduzir o erro médio de previsão, em detrimento de maior variância, ou de incorporar todo o histórico de T e f (menores $\alpha$ e $\beta$ ) para reduzir a variância da previsão, a despeito de um maior erro médio relativo à posição mais recente de $\mathrm{T}$ e $\mathrm{f}$.

\subsection{Impacto da mediana da fração $f$}

Os impactos da fração $f$ dependem da combinação de valores de $\alpha$ e $\beta$. Em linhas gerais, maiores valores de $\alpha / \beta$ implicam aumento na variância e redução no erro médio da abordagem TD se $f \neq 0,50$. A fórmula para $f$ constante fornece valores aproximados para o caso de f estocástico, qualquer que seja o valor de $\alpha$, desde que $\beta=0$. Comparativamente à variância na abordagem $\mathrm{BU}$, a variância na abordagem TD tende a ser menor quando $\alpha>\beta$ e $\mathrm{f} \leq 0,50$; indicando que o efeito ancoragem torna-se mais significativo para menores valores de f. A introdução de viés na previsão TD ocorre para valores de $\mathrm{f}$ diferentes de 0,50; sinalizando um trade-off entre níveis de erro médio e de variância na abordagem TD (Tabela 4).

\section{Implicações e conclusões}

Uma primeira implicação está relacionada aos resultados gerados pela fórmula para f constante para 
Tabela 3. Resultados do experimento com $\mathrm{k}$.

\begin{tabular}{|c|c|c|c|c|c|c|c|}
\hline \multicolumn{2}{|c|}{ f estocástico } & \multicolumn{2}{|c|}{$k=0,5$} & \multicolumn{2}{|c|}{$k=1$} & \multicolumn{2}{|c|}{$\mathbf{k}=\mathbf{2}$} \\
\hline$\alpha$ & $\beta$ & E(EA) & $\mathbf{V}(\mathbf{E A})$ & $\mathbf{E}(\mathbf{E A})$ & $\mathbf{V}(\mathbf{E A})$ & $\mathbf{E}(\mathbf{E A})$ & $\mathbf{V}(\mathbf{E A})$ \\
\hline \multirow[t]{5}{*}{-} & - & 0,782 & 400,000 & - & 400,000 & $(3,640)$ & 400,000 \\
\hline & 0,25 & 0,783 & 419,000 & $(0,002)$ & 430,470 & $(3,659)$ & 506,160 \\
\hline & 0,50 & 0,782 & 444,350 & $(0,002)$ & 471,210 & $(3,635)$ & 639,190 \\
\hline & 0,75 & 0,779 & 479,990 & $(0,005)$ & 528,550 & $(3,635)$ & 834,900 \\
\hline & 1,00 & 0,781 & 533,240 & 0,003 & 613,000 & $(3,622)$ & $1.109,540$ \\
\hline \multirow[t]{5}{*}{0,25} & - & 0,778 & 417,580 & 0,001 & 428,570 & $(3,708)$ & 476,820 \\
\hline & 0,25 & 0,667 & 418,510 & $(0,002)$ & 458,630 & $(3,125)$ & 567,590 \\
\hline & 0,50 & 0,625 & 491,920 & 0,002 & 498,690 & $(2,904)$ & 549,190 \\
\hline & 0,75 & 0,600 & 531,770 & 0,002 & 554,060 & $(2,789)$ & 708,630 \\
\hline & 1,00 & 0,582 & 587,490 & 0,003 & 637,630 & $(2,707)$ & 940,660 \\
\hline \multirow[t]{5}{*}{0,50} & - & 0,781 & 441,020 & $(0,022)$ & 466,700 & $(3,649)$ & 579,050 \\
\hline & 0,25 & 0,629 & 490,420 & $(0,003)$ & 496,600 & $(2,924)$ & 529,130 \\
\hline & 0,50 & 0,521 & 535,450 & $(0,001)$ & 535,530 & $(2,421)$ & 547,570 \\
\hline & 0,75 & 0,447 & 584,140 & 0,001 & 590,210 & $(2,071)$ & 635,860 \\
\hline & 1,00 & 0,391 & 646,340 & $(0,000)$ & 672,540 & $(1,820)$ & 824,340 \\
\hline \multirow[t]{5}{*}{0,75} & - & 0,782 & 473,830 & $(0,004)$ & 520,010 & $(3,666)$ & 722,400 \\
\hline & 0,25 & 0,600 & 527,670 & $(0,004)$ & 550,300 & $(2,786)$ & 654,560 \\
\hline & 0,50 & 0,446 & 581,940 & $(0,000)$ & 588,520 & $(2,074)$ & 622,720 \\
\hline & 0,75 & 0,312 & 641,940 & $(0,001)$ & 642,060 & $(1,458)$ & 649,070 \\
\hline & 1,00 & 0,194 & 715,350 & $(0,001)$ & 722,880 & $(0,911)$ & 764,330 \\
\hline \multirow[t]{5}{*}{1,00} & - & 0,781 & 523,060 & 0,005 & 599,980 & $(3,716)$ & 937,860 \\
\hline & 0,25 & 0,585 & 579,580 & 0,002 & 630,010 & $(2,724)$ & 865,500 \\
\hline & 0,50 & 0,389 & 641,270 & $(0,001)$ & 668,530 & $(1,820)$ & 800,490 \\
\hline & 0,75 & 0,195 & 712,010 & 0,001 & 720,710 & $(0,921)$ & 767,850 \\
\hline & 1,00 & - & 800,020 & - & 800,000 & - & 799,810 \\
\hline
\end{tabular}

Valores negativos são indicados por parênteses.

a variância do erro na abordagem $\mathrm{TD}$, que é dada por $\mathrm{V}(\mathrm{A})+\mathrm{a}^{*} \mathrm{~V}(\mathrm{~T}) /(2-\mathrm{a})$, e sua utilidade para avaliar os casos em que f é estocástico. Para qualquer valor de $\alpha$, desde que $\beta=0$, ela fornece valores exatos para os níveis $\mathrm{f}=0,50$ e $\mathrm{k}=1$ quando a correlação é nula e para quaisquer níveis de $\rho \mathrm{AB}$ quando $\mathrm{f}=0,50 \mathrm{e} \mathrm{k}=1$. Já para $\alpha$ pequeno $(<0,25)$ e $\beta=0$, ela é uma boa aproximação para os casos em que $\mathrm{k}<1$ e $\mathrm{f} \neq 0,50$ e a correlação é nula. Por boa aproximação deve ser entendido um desvio médio inferior a $0,50 \%$ entre os valores simulados e os valores reais.

Nesse sentido, a Equação (3) pode ser utilizada com precisão para determinar os valores críticos de $\mathrm{k}$ para a variância dos erros de previsão entre as duas abordagens quando fé estocástico, desde que $\beta=0$ e $\alpha<0,25$, considerando-se apenas os resultados para $\mathrm{k} \leq 1$. Deve ser notado que esse valor de $\mathrm{k}$ crítico não depende de $\alpha$. Em outras palavras, em primeiro lugar é determinada a abordagem de previsão de menor variância (de acordo com $\rho A B$ e f) e, posteriormente, é efetuada a busca de $\alpha$ ou a decisão de se utilizar um valor de compromisso como 0,10 ; conforme sugerido por Silver et al. (2002).
Uma segunda implicação está relacionada ao viés ou erro médio de previsão presente na abordagem TD quando $\mathrm{f} \neq 0,50$ e $\mathrm{k} \neq 1$. Se $\alpha$ é pequeno e $\beta=0$, esse viés é igual a $\operatorname{COV}(\mathrm{T}, \mathrm{f})$ (cf. Equações (9) e (A1.10) do Anexo 1). Considerando-se que o viés esperado na abordagem BU é zero, esse é aparentemente um ponto desfavorável à abordagem TD no que diz respeito ao seu uso para gerar previsões ou estimativas pontuais de vendas.

Entretanto, dois aspectos devem ser considerados. Primeiramente, se o erro médio de previsão é conhecido a priori, esse valor pode ser utilizado para corrigir a previsão de vendas, ou seja, para remover o viés e equiparar a abordagem TD à BU. Deve ser lembrado que a covariância entre $\mathrm{T}$ e f pode ser facilmente calculada na planilha Excel e/ou em outros pacotes estatísticos. O segundo aspecto está relacionado ao uso ou à decisão que será feita com a previsão. Se a decisão estiver relacionada ao dimensionamento de estoques de segurança, é importante conhecer não apenas o valor esperado da previsão de vendas, mas também a variância do seu erro.

Nesse ponto, cabe comentar as implicações associadas ao dimensionamento de estoques de segurança, relativas ao trade-off entre níveis médios de previsão e de variância 
Tabela 4. Resultados do experimento com $\mathrm{f}$.

\begin{tabular}{|c|c|c|c|c|c|c|c|}
\hline \multicolumn{2}{|c|}{ f estocástico } & \multicolumn{2}{|c|}{$f=0,25$} & \multicolumn{2}{|c|}{$f=0,50$} & \multicolumn{2}{|c|}{$f=0,75$} \\
\hline$\alpha$ & $\beta$ & $\mathbf{E}(\mathbf{E A})$ & $\mathbf{V}(\mathbf{E A})$ & $\mathbf{E}(\mathbf{E A})$ & V(EA) & $\mathbf{E}(\mathbf{E A})$ & $\mathbf{V}(\mathbf{E A})$ \\
\hline \multirow[t]{5}{*}{-} & - & 1,773 & 400,000 & - & 400,000 & $(0,507)$ & 400,000 \\
\hline & 0,25 & 1,776 & 445,760 & $(0,002)$ & 430,470 & $(0,507)$ & 436,370 \\
\hline & 0,50 & 1,777 & 506,780 & $(0,002)$ & 471,210 & $(0,508)$ & 485,240 \\
\hline & 0,75 & 1,772 & 590,650 & $(0,005)$ & 528,550 & $(0,508)$ & 553,270 \\
\hline & 1,00 & 1,765 & 718,110 & 0,003 & 613,000 & $(0,507)$ & 655,040 \\
\hline \multirow[t]{5}{*}{0,25} & - & 1,773 & 406,400 & 0,001 & 428,570 & $(0,510)$ & 464,500 \\
\hline & 0,25 & 1,512 & 465,480 & $(0,002)$ & 458,630 & $(0,437)$ & 457,410 \\
\hline & 0,50 & 1,414 & 528,550 & 0,002 & 498,690 & $(0,408)$ & 488,380 \\
\hline & 0,75 & 1,367 & 611,510 & 0,002 & 554,060 & $(0,390)$ & 546,620 \\
\hline & 1,00 & 1,327 & 733,060 & 0,003 & 637,630 & $(0,378)$ & 640,740 \\
\hline \multirow[t]{5}{*}{0,50} & - & 1,771 & 414,940 & $(0,022)$ & 466,700 & $(0,509)$ & 550,510 \\
\hline & 0,25 & 1,442 & 479,070 & $(0,003)$ & 496,600 & $(0,407)$ & 526,330 \\
\hline & 0,50 & 1,182 & 546,560 & $(0,001)$ & 535,530 & $(0,337)$ & 533,300 \\
\hline & 0,75 & 1,006 & 630,050 & 0,001 & 590,210 & $(0,292)$ & 572,340 \\
\hline & 1,00 & 0,880 & 749,150 & $(0,000)$ & 672,540 & $(0,255)$ & 652,180 \\
\hline \multirow[t]{5}{*}{0,75} & - & 1,773 & 426,890 & $(0,004)$ & 520,010 & $(0,509)$ & 670,920 \\
\hline & 0,25 & 1,350 & 493,120 & $(0,004)$ & 550,300 & $(0,393)$ & 637,640 \\
\hline & 0,50 & 1,016 & 564,650 & $(0,000)$ & 588,520 & $(0,299)$ & 625,240 \\
\hline & 0,75 & 0,711 & 650,720 & $(0,001)$ & 642,060 & $(0,203)$ & 640,820 \\
\hline & 1,00 & 0,441 & 770,180 & $(0,001)$ & 722,880 & $(0,126)$ & 695,040 \\
\hline \multirow[t]{5}{*}{1,00} & - & 1,770 & 444,830 & 0,005 & 599,980 & $(0,509)$ & 851,330 \\
\hline & 0,25 & 1,329 & 512,770 & 0,002 & 630,010 & $(0,381)$ & 812,110 \\
\hline & 0,50 & 0,891 & 586,240 & $(0,001)$ & 668,530 & $(0,253)$ & 784,450 \\
\hline & 0,75 & 0,440 & 675,460 & 0,001 & 720,710 & $(0,128)$ & 775,070 \\
\hline & 1,00 & 0,000 & 799,270 & - & 800,000 & $(0,000)$ & 799,970 \\
\hline
\end{tabular}

Valores negativos são indicados por parênteses.

do erro na abordagem TD, quando $\mathrm{f} \neq 0,50, \mathrm{k} \neq 1$ e a correlação é nula. Existe nesses casos a escolha entre previsões de vendas mais precisas e maior variância (maiores valores de $\alpha$ ) ou de previsões de vendas menos acuradas e menor variância do erro (menores valores de $\alpha$ ).

$O$ valor crítico de $\alpha$, que equilibra o estoque de segurança nas abordagens TD e BU ( $\alpha$ pequeno e $\beta=0$ ), é dado pela Equação 21 (cf. Anexo 3):

$$
\begin{aligned}
& \alpha=\left[2 * \mathrm{~V}(\mathrm{TR}) * \mu \mathrm{T}^{2} * \mathrm{E}(\mathrm{EA}) *(2 * \mu \mathrm{A}-\mathrm{E}(\mathrm{EA}))\right] / \\
& {\left[-\mathrm{TR} * \mathrm{~V}(\mathrm{~A}) * \mu \mathrm{T}^{2}+\mathrm{TR} * \mathrm{~V}(\mathrm{~T}) * \mu \mathrm{A}^{2}-\right.} \\
& -2 * \mathrm{TR} * \mathrm{~V}(\mathrm{~T}) * \mu \mathrm{A} * \mathrm{E}(\mathrm{EA})+\mathrm{TR} * \mathrm{~V}(\mathrm{~T}) * \mathrm{E}(\mathrm{EA})^{2}- \\
& \left.-\mathrm{V}(\mathrm{TR}) * \mu \mathrm{T}^{2} * \mathrm{E}(\mathrm{EA})^{2}+2 * \mathrm{~V}(\mathrm{TR}) * \mu \mathrm{T}^{2} * \mu \mathrm{A} * \mathrm{E}(\mathrm{EA})\right]
\end{aligned}
$$

Sendo que, TR é o tempo de resposta médio, oTR é o desvio padrão do tempo de resposta e V(TR) é a variância do tempo de resposta. Se $\alpha>\alpha$ crítico, um menor estoque de segurança é obtido através da abordagem TD e se $\alpha<\alpha$ crítico, através da BU. Supõe-se que o valor de $\alpha$ seja um valor de compromisso como 0,10 , conforme sugerido por Silver, Pyke e Peterson (2002).

$\mathrm{Se} V(\mathrm{TR})=0, \alpha$ crítico $=0$, ou seja, o nível de estoque de segurança depende diretamente da variância das abordagens TD e BU. Nesse caso, a Equação 3 pode ser utilizada para avaliar qual abordagem de previsão gera menor estoque de segurança. Caso V(TR) $>0, \alpha$ crítico assume valores diferentes de zero, sendo conveniente arredondar valores negativos de $\alpha$ crítico para zero. Nesses casos em que há arredondamento, a abordagem TD é preferível à BU para todos os valores de $\alpha$. Na Figura 2 é apresentado o comportamento de $\alpha$ crítico para diferentes combinações de TR e $\sigma$ TR. Percebe-se que maiores valores de $\sigma \mathrm{TR}$, paralelamente a menores valores de TR (ou seja, um maior coeficiente de variação do tempo de resposta), aumentam o valor de $\alpha$ crítico, favorecendo a abordagem BU no dimensionamento de estoques de segurança. Por outro lado, menor a incerteza relativa ao tempo de resposta e maior o tempo de resposta, o valor de $\alpha$ crítico tende a zero, favorecendo a abordagem TD.

A Figura 2 foi gerada com base no erro médio de previsão obtido para $\mathrm{k}=2$ e $\beta=0$ (Tabela 4): $\mathrm{E}(\mathrm{EA})=(3,638)$. Deve ser notado que quando $\mathrm{E}(\mathrm{EA})=0$, ou seja, $\mathrm{f}=0,50$ e $\mathrm{k}=1$, não existe viés e $\alpha$ crítico $=0$, favorecendo a abordagem TD no dimensionamento de estoques de segurança. Se $\mathrm{f} \neq 0,50$ e $\mathrm{k}>1$, os vieses na previsão TD acabam por favorecer a abordagem BU. 


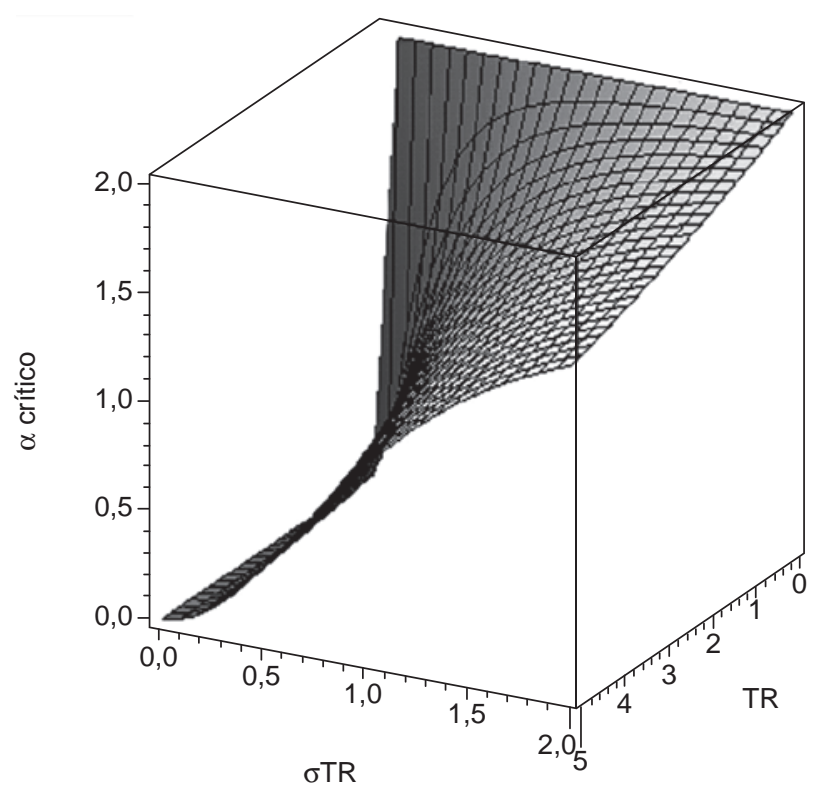

Figura 2. Comportamento de $\alpha$ crítico.

Análises feitas para os dados de todos os experimentos apontam redução média da variância da ordem de $2,4 \%$ e de $3,2 \%$ nos estoques de segurança, considerando-se $\alpha=0,10, \mathrm{TR}=2,5$ e $\sigma \mathrm{TR}=1$.
Finalmente, a última implicação está relacionada a quando deve-se adotar a abordagem TD ou BU em previsão de vendas, sintetizando a discussão precedente para $\alpha<0,25$ e $\beta=0$. A abordagem TD é preferível em termos de variância do erro de previsão quando são verificados, simultaneamente, o efeito ancoragem e o efeito portfólio, ou seja, menor participação do produto nas vendas totais e menor correlação do produto com as vendas agregadas dos demais itens. Esses valores podem ser compensados até certo ponto pela participação da variância do produto na variância total. Ou seja, a abordagem TD pode ser preferível para produtos com grande participação nas vendas e correlação positiva com as vendas dos demais produtos, desde que sua variância seja suficientemente grande.

Se o tempo de resposta é constante, as condições acima indicam que a abordagem TD é preferível para o dimensionamento de estoques de segurança. No entanto, em condições que o tempo de resposta apresenta variabilidade considerável, paralelamente aos casos em que a previsão na abordagem TD possui viés negativo $(\mathrm{k}>1 \mathrm{e}$ $\mathrm{f} \neq 0,50)$, é preferível adotar a abordagem BU.

\section{Top-down or bottom-up forecasting? impact on error and safety inventory levels}

\section{Abstract}

The operations literature remains inconclusive about the most adequate forecasting approach - top-down or bottom-up. This paper presents analytical results for mean values and variances of forecasting errors with exponential smoothing under these approaches. The share of the series in the total aggregate data is considered stochastic. Based on the generation of random numbers, two major impacts are confirmed on the variance of forecasting error, which were presented in previous papers, namely, portfolio and anchorage effects. The impacts are also evaluated in terms of forecasting bias and safety inventory levels.

Keywords: Forecasting approach. Exponential smoothing. Forecasting errors. Safety inventories.

\section{Referências bibliográficas}

BOHRNSTEDT, G.; GOLDBERGER, A. S. On the exact covariance of products of random variables. American Statistical Association Journal, v.64, n.328, p. 1439-1442, dec. 1969.

CHATFIELD, C.; KOEHLER, A. B.; SNYDER, R. D. A new look at models for exponential smoothing. The Statistician, v. 50, n. 2, p. 147-159, 2001.
GELLY, P. Managing bottom-up and top-down approaches: ocean spray's experiences. The Journal of Business Forecasting, v.18, n.4, p. 3-6, Winter 1999.

GIJBELS, I.; POPE, A.; WAND, M. P. Understanding exponential smoothing via kernel regression. Journal of the Royal Statistician Society, v. 61, n. 1, p. 39-50, 1999.

GOODMAN, L. A. On the exact variance of products. American Statistical Association Journal, v.55, n.292, p. 708-713, dec. 1960. 
GORDON, T. P.; MORRIS, J. S.; DANGERFIELD, B. J. Top-down or bottom-up: which is the best approach to forecasting? The Journal of Business Forecasting, v.27, n.3, p.13-16, fall 1997.

GREENE, J. H. Production and inventory control handbook. New York: McGraw-Hill, 1997. p. 658

JAIN, C. How to determine the approach to forecasting. The Journal of Business Forecasting, v.14, p. 2, summer 1995.

KAHN, K. B. Revisiting top-down versus bottom-up forecasting. The Journal of Business Forecasting, v.17, n.2, p. 14-19, summer 1998.

KENNEY, J.; KEEPING, E. Mathematics of statistics - part two. Princeton: Van Nostrand, 1951.p. 429

LAPIDE, L. A simple view of top-down versus bottom-up forecasting. The Journal of Business Forecasting, v.17, n.2, p. 28-29, summer 1998 .

MENTZER, J. T.; KRISHNAN, R. The effect of the assumption of normality on inventory control/customer Service. Journal of Business Logistics, Oak Brooks, v. 6, n. 1, p. 101-120, 1988.

SCHWARZKOPF, A. B.; TERSINE, R. J.; MORRIS, J. S. Top-down versus bottom-up forecasting strategies. International Journal of Production Research, v. 26, n. 11, p. 1833-1843, 1988.
SILVER, E. A.; PETERSON, R. Decision systems for inventory management and production planning. New York: Wiley, 1985. p. 736

SILVER, E. A.; PYKE, D.; PETERSON, R. Decision systems for inventory management and production planning and scheduling. New York: Wiley, 2002. p. 754

WANKE, P. Impacto das abordagens top-down e bottom-up na variância do erro de previsão com amortecimento exponencial simples. In: WANKE, P.; FERREIRA, L. J. Previsão de vendas. São Paulo: Editora Atlas, 2006. p. 260

WEISSTEIN, E. W. Cauchy distribution. In: MathWorld - A Wolfram Web Resource. Disponível em: <http://mathworld. wolfram.com/cauchydistribution.html>, 1999. Acesso em: 6 set. 2006.

ZINN, W.; LEVY, M.; BOWERSOX, D. J. Measuring the effect of inventory centralization/decentralization. Journal of Business Logistics, Oak Brooks, v. 10, n. 2, p. 1-14, 1989.

ZWILLINGER, D.; KOKOSA, S. Standard probability and statistics tables and formulae. New York: Chapman\&Hall, 2000. p. 554

\section{Anexo 1. Valores exatos e aproximados de $E(P A)$ e $V(E A)$ para pequenos valores de $\alpha$ e $\beta$}

Por exemplo, COV(PT,Pf) é dado por:

$$
\begin{aligned}
& \operatorname{COV}(\mathrm{PT}, \mathrm{Pf})=\operatorname{COV}\left[\left(\alpha^{*} \mathrm{~T}+(1-\alpha) * \mathrm{PT}\right),(\beta * \mathrm{f}+(1-\beta) * \mathrm{Pf})\right] \\
& \operatorname{COV}(\mathrm{PT}, \mathrm{Pf})=\alpha^{*} \beta^{*} \operatorname{COV}(\mathrm{T}, \mathrm{f})+(1-\alpha) *(1-\beta) * \operatorname{COV}(\mathrm{PT}, \mathrm{Pf})+\alpha^{*}(1-\beta) * \operatorname{COV}(\mathrm{T}, \mathrm{Pf})+(1-\alpha)^{*} \beta^{*} \operatorname{COV}(\mathrm{PT}, \mathrm{f})
\end{aligned}
$$

Dado que $\operatorname{COV}(\mathrm{PT}, \mathrm{f})$ e $\operatorname{COV}(\mathrm{T}, \mathrm{Pf})$ são aproximadamente iguais a zero, então:

$$
\operatorname{COV}(\mathrm{PT}, \mathrm{Pf}) \approx \frac{\alpha * \beta * \operatorname{COV}(\mathrm{T}, \mathrm{f})}{1-(1-\alpha) *(1-\beta)}
$$

Já os termos $\operatorname{COV}\left(\Delta \mathrm{TP}^{2}, \Delta \mathrm{Pf}^{2}\right), \operatorname{COV}\left(\Delta \mathrm{TP}^{2}, \Delta \mathrm{Pf}\right)$ e $\operatorname{COV}\left(\Delta \mathrm{TP}, \Delta \mathrm{Pf}^{2}\right)$ são pequenos e aproximadamente iguais a zero quando $\alpha$ e $\beta$ são menores que 0,25 , implicando que a variância do erro de previsão de A na abordagem TD pode ser aproximada por:

$$
\begin{aligned}
& \mathrm{V}(\mathrm{EA}) \approx \mathrm{V}(\mathrm{A})+\frac{\beta}{2-\beta} * \mathrm{E}(\mathrm{T})^{2} * \mathrm{~V}(\mathrm{f})+\frac{\alpha}{2-\alpha} * \mathrm{E}(\mathrm{f})^{2} * \mathrm{~V}(\mathrm{~T})+\frac{\alpha * \beta}{(2-\alpha)^{*}(2-\beta)} * \mathrm{~V}(\mathrm{~T}) * \mathrm{~V}(\mathrm{f})+ \\
& +\frac{2 * \mathrm{E}(\mathrm{f}) * \mathrm{E}(\mathrm{T}) * \alpha * \beta}{1-(1-\alpha) *(1-\beta)} * \mathrm{COV}(\mathrm{T}, \mathrm{f})-\left(\frac{\alpha * \beta}{1-(1-\alpha) *(1-\beta)}\right)^{2} * \operatorname{COV}(\mathrm{T}, \mathrm{f})^{2}
\end{aligned}
$$

Já o valor esperado da previsão de vendas pode ser aproximado por:

$$
\mathrm{E}(\mathrm{PA}) \approx \mathrm{E}(\mathrm{T}) * \mathrm{E}(\mathrm{f})+\frac{\alpha * \beta}{1-(1-\alpha) *(1-\beta)} * \operatorname{COV}(\mathrm{T}, \mathrm{f})
$$

Sendo $V(A), E(T), V(T), E(f), V(f)$ e COV(T,f) determinados diretamente das séries de vendas, o que pode ser facilmente obtido em planilha MS-Excel. Finalmente, para o caso particular em que $\beta=0, \operatorname{COV}\left(\Delta \mathrm{TP}^{2}, \Delta \mathrm{Pf}^{2}\right), \operatorname{COV}\left(\Delta \mathrm{TP}^{2}, \Delta \mathrm{Pf}\right)$ e $\operatorname{COV}\left(\Delta \mathrm{TP}, \Delta \mathrm{Pf}^{2}\right)$ são iguais a zero e:

$$
\begin{aligned}
& \mathrm{V}(\mathrm{EA})=\mathrm{V}(\mathrm{A})+\alpha /(2-\alpha) * \mathrm{E}(\mathrm{f}) * \mathrm{~V}(\mathrm{~T}) \\
& \mathrm{E}(\mathrm{PA})=\mathrm{E}(\mathrm{T}) * \mathrm{E}(\mathrm{f})
\end{aligned}
$$

mas: 


$$
\begin{aligned}
& \mathrm{E}(\mathrm{EA})=\operatorname{COV}(\mathrm{T}, \mathrm{f})-\operatorname{COV}(\mathrm{PT}, \mathrm{Pf}) \\
& \mathrm{E}(\mathrm{EA})=\left(2^{*} \alpha^{*} \beta-\alpha-\beta\right) /(\alpha * \beta-\alpha-\beta) * \operatorname{COV}(\mathrm{T}, \mathrm{f}) \\
& \mathrm{E}(\mathrm{EA})=\operatorname{COV}(\mathrm{T}, \mathrm{f}) \\
& \mathrm{e}:
\end{aligned}
$$

$$
\operatorname{COV}(\mathrm{T}, \mathrm{f})=\mathrm{E}(\mathrm{A})-\mathrm{E}(\mathrm{T}) * \mathrm{E}(\mathrm{f})
$$

Logo:

$$
\mathrm{E}(\mathrm{f})=\frac{\mu \mathrm{A}-\mathrm{E}(\mathrm{EA})}{\mu \mathrm{T}}
$$

então:

$$
\begin{aligned}
& \mathrm{V}(\mathrm{EA})=\mathrm{V}(\mathrm{A})+\frac{\alpha}{2-\alpha} *\left(\frac{\mu \mathrm{A}-\mathrm{E}(\mathrm{EA})}{\mu \mathrm{T}}\right)^{2} * \mathrm{~V}(\mathrm{~T}) \\
& \mathrm{E}(\mathrm{PA})=\mu \mathrm{A}-\mathrm{E}(\mathrm{EA})
\end{aligned}
$$

\section{Anexo 2. Valor esperado e variância de $f$}

Seja a expressão da covariância entre $\mathrm{T}$ e f:

$$
\begin{aligned}
& \operatorname{COV}(\mathrm{T}, \mathrm{f})=\mathrm{E}(\mathrm{T} * \mathrm{f})-\mathrm{E}(\mathrm{T}) * \mathrm{E}(\mathrm{f}) \\
& \rho \mathrm{Tf} f^{*} \sigma \mathrm{T}^{*} \sigma \mathrm{f}=\mathrm{E}(\mathrm{A})-\mathrm{E}(\mathrm{T}) * \mathrm{Ef}
\end{aligned}
$$

Em que $\rho \mathrm{Tf}$ é o coeficiente de correlação linear entre T e f, $\sigma \mathrm{T}$ é o desvio padrão de $\mathrm{T}$ e of é o desvio padrão de f. Dividindo-se ambos os lados por $\sigma \mathrm{T}$, tem-se a reta:

$$
\rho \mathrm{Tf} * \sigma \mathrm{f}=\frac{1}{\mathrm{CVa}^{*} \sqrt{\Delta}}-\frac{\mathrm{f}}{\mathrm{CVt}}
$$

Sendo CVt o coeficiente de variação de T; CVa o coeficiente de variação de $A$ e $D=k^{2}+2 * k^{*} \rho A B+1$. Nessa reta, a relação entre $\rho T f^{*} \sigma f$ é estabelecida pelo coeficiente angular $-1 / \mathrm{CV}$ t e pelo coeficiente linear $1 /(\mathrm{CVa} * \sqrt{\Delta})$, conforme é ilustrado na Figura 1.

Variações infinitesimais no ângulo $\theta$ farão com que o valor esperado de $\mathrm{f}(\mathrm{E}(\mathrm{f})$ ) se desloque de sua mediana teórica $(A / T)$ na reta apresentada na Figura 1. Correlações positivas entre $T$ e f implicarão que $E(f)<A / T$, correlações negativas implicarão o contrário. Percebe-se que quando $\rho \mathrm{Tf}=0$ ou $\sigma \mathrm{f}=0 ; \mathrm{E}(\mathrm{f})=\mathrm{A} / \mathrm{T}$.

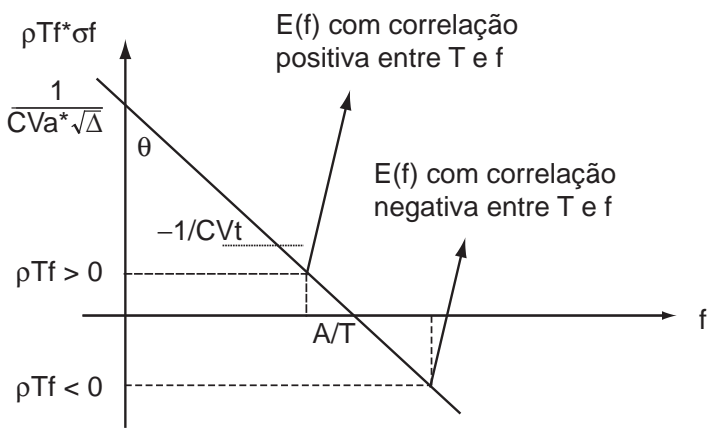

Figura 1. Relação entre $\rho \mathrm{Tf}$ * $\sigma$ e f. 
Então:

$$
\begin{aligned}
& \tan \theta=\frac{\mathrm{f}}{\frac{1}{\mathrm{CVa} * \sqrt{\Delta}}-\frac{\mathrm{f}}{\mathrm{CVt}}}=\frac{\mathrm{CVt} * \mathrm{CVa}^{*} \sqrt{\Delta} * \mathrm{f}}{\mathrm{CVt}-\mathrm{CVa}^{*} \sqrt{\Delta} * \mathrm{f}} \\
& \theta=\arctan \left(\frac{C V t * C V a * \sqrt{\Delta} * f}{C V t-C V a * \sqrt{\Delta} * f}\right) \\
& \mathrm{d} \theta=\frac{\frac{\mathrm{CVt} * \mathrm{CVa} * \sqrt{\Delta}}{\mathrm{CVt}-\mathrm{CVa} * \sqrt{\Delta} * \mathrm{f}}+\frac{\mathrm{CVt} * \mathrm{CVa}^{2} * \Delta * \mathrm{f}}{(\mathrm{CVt}-\mathrm{CVa} * \sqrt{\Delta} * \mathrm{f})^{2}}}{1+\frac{\mathrm{CVt} \mathrm{CVa}^{2} * \Delta * \mathrm{f}^{2}}{(\mathrm{CVt}-\mathrm{CVa} * \sqrt{\Delta} * \mathrm{f})^{2}}}
\end{aligned}
$$
Normalizando para todos os ângulos dados que $\int_{-\pi / 2}^{+\pi / 2} d \theta=1$, tem-se que a função densidade de probabilidade de $\mathrm{f}$
(p(f)) é dada por:

$$
\mathrm{p}(\mathrm{f})=\frac{\frac{\mathrm{CVt} * \mathrm{CVa}^{*} \sqrt{\Delta}}{\mathrm{CVt}-\mathrm{CVa}^{*} \sqrt{\Delta} * \mathrm{f}}+\frac{\mathrm{CVt} * \mathrm{CVa}^{2} * \Delta * \mathrm{f}}{(\mathrm{CVt}-\mathrm{CVa} * \sqrt{\Delta} * \mathrm{f})^{2}}}{\left(1+\frac{\mathrm{CVt}^{2} * \mathrm{CVa}^{2} * \Delta * \mathrm{f}^{2}}{\left(\mathrm{CVt}-\mathrm{CVa}^{*} \sqrt{\Delta} * \mathrm{f}\right)^{2}}\right) *\left(\ln \left(\frac{-\mathrm{I} * \sqrt{\Delta} * \mathrm{CVa}^{*}\left(1+\mathrm{CVt}^{2}\right)}{\mathrm{CVt}^{2}}\right) * \mathrm{I}-\ln \left(\frac{+\mathrm{I}^{*} \sqrt{\Delta} * \mathrm{CVa}^{*}\left(1+\mathrm{CVt}^{2}\right)}{\mathrm{CVt}^{2}}\right) * \mathrm{I}\right)}
$$

Em que I é o número complexo $\sqrt{-1}$. A função de probabilidade acumulada de $\mathrm{f}(\mathrm{F}(\mathrm{f}))$ é:

$$
\begin{aligned}
& \mathrm{F}(\mathrm{f})=\int_{\mathrm{f}} \mathrm{p}(\mathrm{f}) \mathrm{df} \\
& \mathrm{F}(\mathrm{f})=\frac{\arctan \left(\frac{2 *\left(\mathrm{CVa}^{2} * \Delta+\mathrm{CVt}^{2} * \mathrm{CVa}^{2} * \Delta\right) * \mathrm{f}-2 * \mathrm{CVt} * \mathrm{CVa} * \sqrt{\Delta}}{2 * \sqrt{\Delta} * \mathrm{CVt}^{2} * \mathrm{CVa}}\right)}{\ln \left(\frac{-\mathrm{I} * \sqrt{\Delta} * \mathrm{CVa}^{2}\left(1+\mathrm{CVt}^{2}\right)}{\mathrm{CVt}^{2}}\right) * \mathrm{I}-\ln \left(\frac{+\mathrm{I} * \sqrt{\Delta} * \mathrm{CVa}^{2}\left(1+\mathrm{CVt}^{2}\right)}{\mathrm{CVt}^{2}}\right) * \mathrm{I}}
\end{aligned}
$$

Sendo possível verificar que:

$$
\begin{aligned}
& F(f)=\int_{-\infty}^{+\infty} p(f) d f=1 \\
& F(f)=\int_{0}^{A / T} p(f) d f=1 / 2
\end{aligned}
$$

Ou seja, A/T é a mediana teórica de f. No entanto, não são definidas as integrais para:

$E(f)=\int_{-\infty}^{+\infty} p(f) * f d f$ e $V(f)=\int_{-\infty}^{+\infty} p(f) *(f-E(f))^{2} d f$, dado que elas divergem para os momentos de ordem maior que 1.

Dessa forma, não há como demonstrar analiticamente, através de diferenciação, para quais valores de $\alpha, \beta, \mathrm{A}, \mathrm{T}$ e f a abordagem TD apresenta menor média e/ou variância no erro de previsão que a abordagem BU. Por isso, a necessidade de conduzir experimentos de simulação.

\section{Anexo 3. Determinação de $\alpha$ crítico}

Sejam ES(TD) e ES(BU), respectivamente, os estoques de segurança para as abordagens TD e BU. Sendo $\gamma$ o fator de serviço para o erro de previsão no tempo de resposta com distribuição normal, $\alpha$ crítico é obtido igualando-se (A3.1) e (A3.2).

$$
\begin{aligned}
& \mathrm{ES}(\mathrm{TD})=\gamma * \sqrt{\mathrm{TR} * \mathrm{~V}(\mathrm{EA})+\sigma \mathrm{TR}^{2} *(\mu A-\mathrm{E}(\mathrm{EA}))^{2}} \\
& \mathrm{ES}(\mathrm{BU})=\gamma * \sqrt{\mathrm{TR} * \mathrm{~V}(\mathrm{BU})+\sigma \mathrm{TR}^{2} * \mu \mathrm{A}^{2}}
\end{aligned}
$$




\section{Sobre o autor}

\section{Peter Wanke}

Centro de Estudos em Logística, Instituto COPPEAD de Administração, UFRJ,

Ed. COPPEAD, Cidade Universitária, Ilha do Fundão, CEP 21949-900, Rio de Janeiro, RJ, Brasil, e-mail: peter@coppead.ufr..br 